\title{
Non-resonant magnetic braking on JET and TEXTOR
}

\author{
Y. Sun ${ }^{*}{ }^{1}$ Y. Liang, ${ }^{1}$ H. R. Koslowski, ${ }^{1}$ K.C. Shaing, ${ }^{2,}{ }^{3}$ S. Jachmich, ${ }^{4}$ A. Alfier,${ }^{5}$ \\ O. Asunta, ${ }^{6}$ G. Corrigan, ${ }^{7}$ E. Delabie,${ }^{1}$ C. Giroud,${ }^{7}$ M. P. Gryaznevich, ${ }^{7}$ \\ D. Harting, ${ }^{1}$ T. Hender,${ }^{7}$ E. Nardon, ${ }^{7}$ V. Naulin, ${ }^{8}$ V. Parail,${ }^{7}$ T. Tala,${ }^{9}$ \\ C. Wiegmann, ${ }^{1}$ S. Wiesen, ${ }^{1}$ T. Zhang, ${ }^{1}$ and JET-EFDA contributors ${ }^{\dagger}$ \\ (JET-EFDA, Culham Science Centre, OX14 3DB, Abingdon,UK) \\ ${ }^{1}$ Institute for Energy Research - Plasma Physics, \\ Forschungszentrum Jülich, Association EURATOM-FZJ, \\ Trilateral Euregio Cluster, 52425 Jülich, Germany \\ ${ }^{2}$ Institute for Space, Astrophysical and Plasma Sciences, \\ National Cheng Kung Unversity, Tainan, Taiwan 70101, Republic of China \\ ${ }^{3}$ Engineering Physics Department, University of Wisconsin, Madison, WI 53706, USA \\ ${ }^{4}$ Association EURATOM-Belgian State, \\ Koninklijke Militaire School-Ecole Royale Militaire, B-1000 Brussels, Belgium \\ ${ }^{5}$ Associazione EURATOM-ENEA sulla Fusione, Consorzio RFX Padova, Italy \\ ${ }^{6}$ Association EURATOM-Tekes, Aalto University, \\ P.O.Box 14100 FI-00076 AALTO, Finland \\ ${ }^{7}$ EURATOM-CCFE, Culham Science Centre, Abingdon, OX14 3DB, UK \\ ${ }^{8}$ Association EURATOM-Ris $\phi$ National Laboratory, \\ OPL-128 Ris $\phi$, DK-4000 Roskilde, Denmark \\ ${ }^{9}$ Association EURATOM-Tekes, VTT, \\ P.O. Box 1000, FIN-02044 VTT, Finland
}

\begin{abstract}
The non-resonant magnetic braking effect induced by an NonAxisymmetric Magnetic Perturbation (NAMP) is investigated on JET and TEXTOR. The collionality dependence of the torque induced by the $n=1$ NAMP field is obtained on JET. The observed torque is located mainly in the plasma core (normalized $\rho<0.4$ ). It increases with decreasing collisionality. The calculation shows that it is close to the transition between the $\nu-\sqrt{\nu}$ and the superbanana plateau regimes in the plasma core. The NTV torque is modeled by using the smoothly connnected formula in the collisionless regimes. The calculated collisionality dependence shows the same tendency as the experimental observation due to the resonant particle effect. The strongest NTV torque is also located in the plasma core because of the resonant particle effect. However, the magnitudes of the NTV torque is still about 1-2 orders smaller than the observed ones on JET. There is no obvious braking effect with $m / n=6 / 2$ NAMP generated by the Dynamic Ergodic Divertor (DED) on TEXTOR. The calculated NTV torque on TEXTOR is also very small.
\end{abstract}

\section{Introduction}

The magnetic field of a tokamak is designed to be toroidally symmetric. Realistically, there is always a slight Non-Axisymmetric Magnetic Perturbation (NAMP) due to an intrinsic error field, MagnetoHydroDynamics (MHD) perturbations in the plasma or external magnetic perturbations applied to control Edge Localized Modes (ELMs) $[1,2]$ and Resistive Wall Modes (RWMs) [3].

\footnotetext{
* Email: y.sun@fz-juelich.de

$\dagger$ See the Appendix of F. Romanelli et al., paper OV/1-3, this conference
} 
The magnetic braking effect without mode locking during the application of NAMP has been observed in several tokamak experiments [4-8]. In a low collisionality plasma, breaking of the toroidal symmetry will result in a so-called Neoclassical Toroidal plasma Viscosity (NTV) [9] torque. The NTV torque is a potential explanation of the observed braking effect.

A good agreement between the calculated NTV torque in the $1 / \nu$ regime and the observed one induced by the applied NAMP has been reported from NSTX [4]. Here, $\nu$ is the collisionality. The collisionality dependence of the braking effect on NSTX was also consistent with the NTV torque in the $1 / \nu$ regime [7]. On DIII-D, the calculated NTV damping time in the $\nu$ regime was about two orders longer than the observation [10]. The MHD induced braking effect on MAST was in agreement with the NTV in the $1 / \nu$ regime $[11]$.

A strong non-resonant magnetic braking has been observed in ELM control experiments with $n=1$ NAMP field generated by the Error Field Correction Coils (EFCCs) on JET $[8,12]$. The observed torque was mainly located in the plasma core and it was about half of the Neutral Beam Injection (NBI) torque on JET [8]. The magnitude of the observed torque is between the torque predicted by the NTV theory in the $\nu-\sqrt{\nu}[13]$ and $1 / \nu$ regimes. The NTV torque in the $\nu-\sqrt{\nu}$ is too small to explain the observed torque [8].

The experimental regime in present tokamaks as well as ITER [10] covers all the $1 / \nu$ [9], $\nu-\sqrt{\nu}[13]$ and the superbanana plateau [14] regimes and the transitions between them. Furthermore, particles with different energy are in different collisionality regimes. In order to model the toroidal plasma rotation with NAMP and compare it with the observation, we need to know the NTV solution in the transition regime, as well as in the asymptotic limits of these collisionless regimes.

One approximate analytic general expression of NTV torque has been obtained by smoothly connecting the formula in these different regimes by Shaing et al.[15]. Another general analytic result has been obtained by using the simple Krook collisional operator by Park et al.[16]. Recently, a general solution with the pitch angle scattering collisional operator is obtained by Sun et al. by numerically solving the bounce averaged drift kinetic equation [17]. The numerical results were in a good agreement with the smoothly connected formula, while showed some differences at the transition regimes. It has been shown in [17] that the resonant particle effect [14] is important for a relatively low collisionality and low plasma rotation case. The resonant particle effect can strongly change the NTV torque dependence on the collisionality.

In this paper, the non-resonant magnetic braking effect induced by the NAMP on JET and TEXTOR is investigated and it is compared with the NTV theory. This paper is organized as follows. The experimental observations are presented in section 2. The NTV theory in the collisionless regimes is summarized in setion 3. The NTV torque profiles are calculated and compared with the observations in section 4 , followed by the summary of the main results in section 5 .

\section{Experimental observations}

2.1 Collisionality dependence of the braking effect by $n=1$ NAMP on JET

A density pump out usually occurs in ELM control experiment by the application of a low $n$ NAMP field on JET [12]. Recently, the plasma density drop during the application of NAMP field was compensated by gas puffing (JET pulse 77334 as shown in Fig. 1) or pellet injection (JET pulse 77328, 77331, 77333 and 77337 as shown in Fig. 1). The equilibrium configuration of these pulses were kept the same. The plasma collisionality changed a lot when the plasma density was restored at different levels.

Fig. 1 shows the temporal evolution of the plasma rotation (A), density (B), ion 


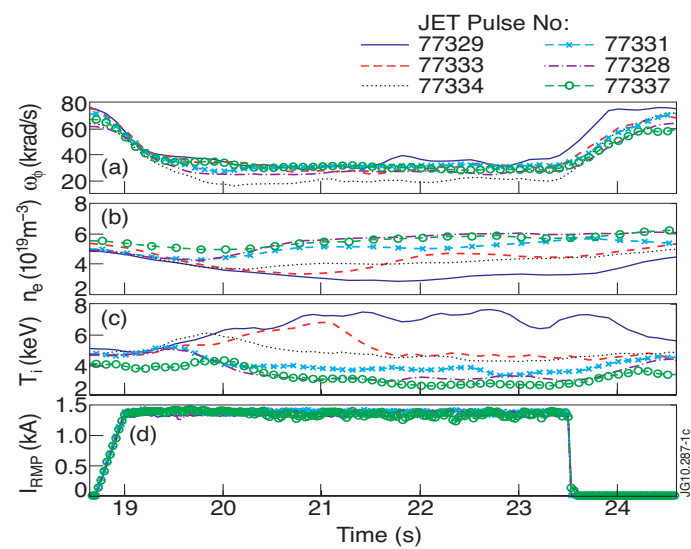

FIG. 1: The temporal evolution of the plasma rotation (A), density (B), ion temperature (C) at $R_{0}=3.0 \mathrm{~m}$ (near the magnetic axis) during the application of $n=1$ NAMP field (D) for a series of pulses where the densities were restored at different levels.

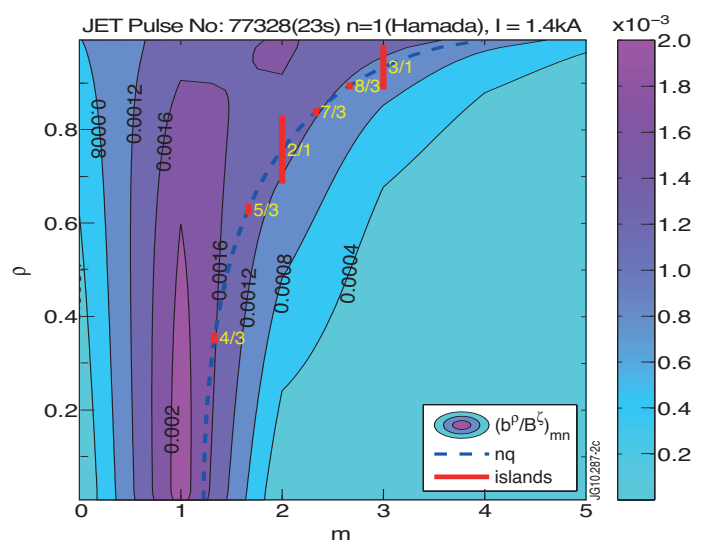

FIG. 2: The strength of the $n=1$ component of the RMP spectrum $\left(b^{\rho} / B^{\zeta}\right)_{m n}$ in Hamada coordinates, the resonance condition $m=n q$ (dashed line) and the locations and widths of the islands (red lines) induced by the NAMP using the vacuum field assumption.

temperature (C) at $R_{0}=3.0 \mathrm{~m}$ (near the magnetic axis) during the application of $n=1$ NAMP field (D) for a series of pulses where the densities were restored at different levels. In these pulses, the toroidal magnetic field strength is $B_{0}=1.8 \mathrm{~T}$, the injected beam power is $10.5 \mathrm{MW}$ and the safety factor $q$ at $\hat{\psi}_{p}=0.95$ is $q_{95}=3.2$. Here, $\hat{\psi}_{p}$ is the normalized poloidal magnetic flux. After the NAMP field is applied, the plasma core rotation for each pulse dramatically reduces to about half of its initial rotation without NAMP. There is no mode locking observed in these pulses.

The strength of the $n=1$ component of the Resonant Magnetic Perturbation (RMP) spectrum $\left(b^{\rho} / B^{\zeta}\right)_{m n}$ in Hamada coordinates of the applied NAMP is shown in the contour plot in Fig. 2. The vertical axis is the radial position and the horizontal axis is the poloidal mode number $m$. The radial coordinate $\rho=\sqrt{\psi_{T} / \pi B_{0}}$ is used in this paper (Normalized $\rho$, with $\rho=1$ at the last close surface, is used in all the figures). Here $\psi_{T}$ is the toroidal magnetic flux and $B_{0}$ is the magnetic field strength at the magnetic axis. The dashed line indicates the resonance condition $m=n q$ at different radii, and the red lines mark the locations and widths of the islands induced by the NAMP using the vacuum field assumption. There is a small $n=3$ harmonic in this coil configuration.

Among these pulses the effective ion collisionalities $\nu_{*, i} \equiv \nu_{i} /\left(\epsilon^{3 / 2} \omega_{t i}\right)$ in the plasma core region are changed by a factor of 6 which is shown in Fig. 3. Here $\nu_{i}$ is the ion collisionality, $\omega_{t i}=v_{t i} / q R_{0}$ is the ion transient frequency and $v_{t i}$ is the ion thermal velocity.

The torque density profiles induced by the $n=1 \mathrm{NAMP}$ at $t=23 \mathrm{~s}$ are calculated by momentum transport analysis [8] and are shown in Fig. 4. The maximum torque is located at the plasma core region, which is similar to previous observation [8].

The obtained torque profiles obviously depend on the collisionality. The collsionality dependence of the total torque inside the $\rho=0.4$ is shown in Fig. 5. The torque increases with decreasing collisionality, which is similar to the dependence observed on NSTX [7].

2.2 Non-resonant magnetic braking experiment with $m / n=6 / 2$ DED on TEXTOR

The non-resonant magnetic braking induced by the Dynamic Ergodic Divertor (DED) [18] in $m / n=6 / 2$ configuration on TEXTOR is investigated. The strength of the $n=2$ 


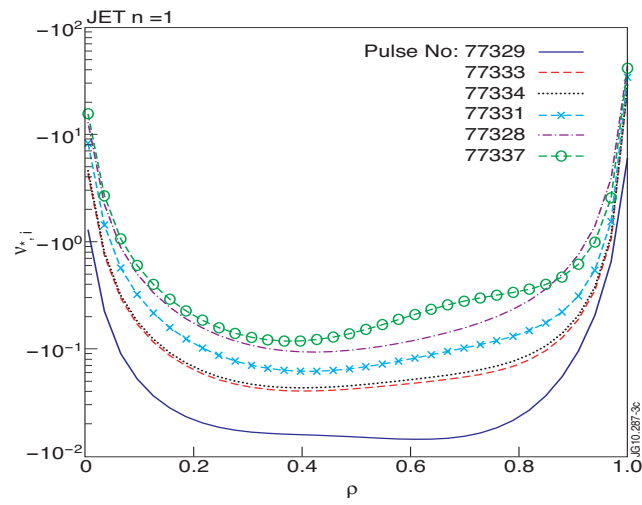

FIG. 3: The profiles of the effective collisionality

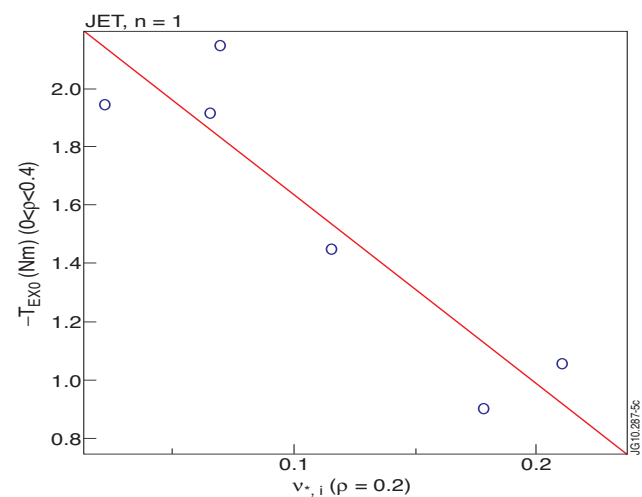

FIG. 5: The observed total torque inside the $\rho=0.4$ surface

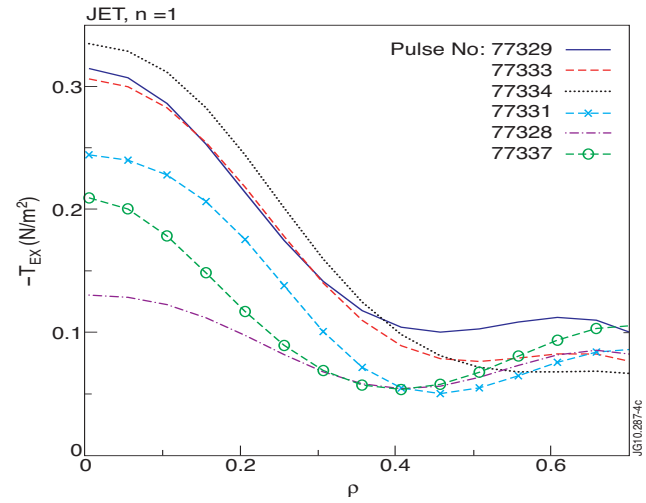

FIG. 4: The observed torque density profiles induced by NAMP

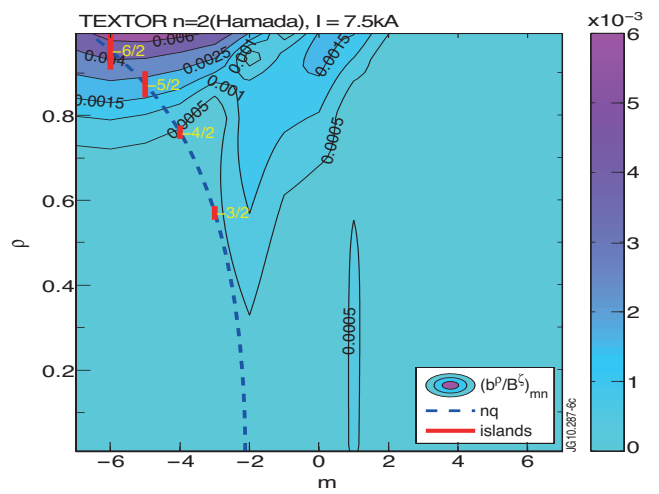

FIG. 6: The RMP spectrum of the $m / n=6 / 2$ DED coil configuration on TEXTOR

component of the RMP spectrum of $m / n=6 / 2$ DED coil configuration is shown in Fig. 6. No obvious braking effect is observed with this coil configuration for both resonant $(\mathrm{m} / \mathrm{n}=-6 / 2$ as shown in the Fig. 6, where '-' is due to convention of the definition of the coordinates) and non-resonant $(\mathrm{m} / \mathrm{n}=6 / 2$, by changing the plasma current orientation, i.e. changing the sign of the $q$ ) pitch cases before the field penetrates.

\section{NTV theory}

The equilibrium magnetic field in tokamaks can be expressed as $\vec{B}_{e q}=\frac{d \psi_{p}}{d \hat{V}} \nabla \hat{V} \times \nabla \alpha=$ $\nabla \phi \times \nabla \psi_{p}+g(\psi) \nabla \phi$, where $2 \pi \psi_{p}$ is the poloidal magnetic flux, $\hat{V} \equiv V /\left(4 \pi^{2}\right), \mathrm{V}$ is the plasma volume enclosed by the flux surface and $\alpha=q \theta-\zeta$ is the drift angle, $\phi$ is the geometric toroidal angle, $g=R B_{t}$ and $B_{t}$ is the toroidal magnetic field. $(\hat{V}, \theta, \zeta)$ are the Hamada coordinates with Jacobin $\mathcal{J}=1$. Therefore, the Jacobin of $(\rho, \theta, \zeta)$ becomes $\mathcal{J}=\hat{V}^{\prime}=\rho B_{0} /\left(g<1 / R^{2}>_{\psi}\right)$, where the prime denotes the derivative over $\rho$ and $<\ldots\rangle_{\psi}$ denotes flux surface average.

The magnetic field strength with NAMP can be written as,

$$
B=B_{0}\left[1-\epsilon \cos \theta-\sum_{n} b_{n}(\theta) e^{i n \alpha}\right]
$$

where $\epsilon \approx r / R$ is the amplitude of the $\cos \theta$ component of the equilibrium field caused by toroidicity, $r$ and $R$ are the minor and major radius, respectively, $b_{n}=\sum_{m} b_{m n} e^{i(m-n q) \theta}$ is the $n^{\text {th }}$ Fourier harmonic of the perturbation field and $b_{m n}$ are the Fourier coefficients of $\delta B / B_{0}$ in $(\theta, \zeta)$ coordinates on the distorted flux surfaces [9]. 
The variation of the magnetic field strength on the distorted flux surface (or the Lagrangian variation) due to the NAMP $\vec{b}$ can be written as $[8,9,19]$,

$$
\delta B=\vec{b} \cdot\left(\vec{B}_{e q} / B_{e q}\right)+\left(\xi^{\rho} \partial_{\rho} B_{e q}+\xi^{\theta} \partial_{\theta} B_{e q}\right)
$$

where $\vec{\xi}$ is the displacement induced by the NAMP. The first term is the so called Eularian part [19]. The NTV theory is based on the existence of closed flux surfaces. From $\vec{B}_{e q} \cdot \nabla \vec{\xi}=\vec{b}$, the spectrum of the displacement can be written as,

$$
\xi_{m n}^{\rho, \theta}=q \frac{\left(b^{\rho, \theta} / B^{\zeta}\right)_{m n}}{m-n q}
$$

There are singularities at the rational surfaces $q_{s}=m / n$, if $\left.\left(b^{\rho, \theta} / B^{\zeta}\right)_{m n}\right|_{q_{s}} \neq 0$, i.e. the existence of island. These singularities can be removed by changing $1 /(m-n q)$ to $(m-n q) /\left[(m-n q)^{2}+\delta_{m n}^{2}\right]$, which is similar to the method used in [19]. The small parameter $\delta_{m n}$ can be chosen as $\delta_{m n}=\left.n w_{m n} q^{\prime}\right|_{q_{s}} / 2$, where $w_{m n}=\left.4 \sqrt{q\left(b^{\rho} / B^{\zeta}\right)_{m n} / n q^{\prime}}\right|_{q_{s}}$ is the island width.

The typical effective collisionality $\nu_{*}$, satisfies $\left(\frac{\delta B / B}{\epsilon}\right)^{3 / 2} \ll \nu_{*} \ll 1$ in most tokamak operational regimes for present tokamaks as well as ITER. The collisionless detrapping/retrapping regime [20] and the superbanana regime [21] are usually not accessible.

The general form of the toroidal NTV torque density can be written as $[15,17]$,

$$
T_{N T V}=-\left\langle R^{2}\right\rangle_{\psi} \tau_{N T V}^{-1} \rho_{i} \omega_{\phi}
$$

where

$$
\begin{gathered}
\tau_{N T V}^{-1}=\left\langle 1 / R^{2}\right\rangle_{\psi} R_{0}^{2} \sum_{j=i, e} \sum_{n} \frac{\sqrt{\epsilon} q^{2} \omega_{t i}^{2}}{2 \sqrt{2} \pi^{3 / 2}}\left|\frac{e_{i}}{e_{j}}\right| \lambda_{1, n}\left(1-\omega_{n c, n} / \omega_{\phi}\right) \\
\lambda_{l, n} \equiv \frac{1}{2} \int_{0}^{\infty} I_{\kappa n}(x)(x-5 / 2)^{l-1} x^{5 / 2} e^{-x} d x \\
\omega_{n c, n}^{j} \equiv q\left(\omega_{\theta}+\omega_{*, j}-\omega_{*, i}+\frac{\lambda_{2, n}}{\lambda_{1, n}} \omega_{* T, j}\right)
\end{gathered}
$$

and $\rho_{j}=N_{j} M_{j}$ and $e_{j}=Z_{j} e_{0}$ are the mass density and electric charge of the $j^{t h}$ kind of particles, respectively, $\omega_{\phi}$ and $\omega_{\theta}$ are the toroidal and poloidal ion rotation angular frequencies. $\omega_{n c}$ is the general form of the so-called neoclassical "offset" rotation $[5,22]$. The diamagnetic frequencies $\omega_{*} \equiv P^{\prime} /\left(\rho B_{0} e N\right)$, and $\omega_{* T} \equiv T^{\prime} /\left(\rho B_{0} e\right) . P$ is the plasma pressure, $T$ is the plasma temperature,

The solution of the pitch angle integral $I_{\kappa n}$ depends on the collisionality regime.

When $\nu_{* d} \equiv \frac{\nu_{d} /(2 \epsilon)}{\left|n \omega_{d \alpha}\right|} \approx \frac{\nu_{d} /(2 \epsilon)}{\left|n q \omega_{E}\right|} \gg 1$, the regime is the so-called $1 / \nu$ regime. The pitch angle integral in this regime can be written as [9],

$$
I_{\kappa n, 1 / \nu}=\frac{I_{1}^{2}}{\nu_{d} /(2 \epsilon)} \int_{0}^{1} 16 K F^{-1}\left|n\left\langle\left[\kappa^{2}-\sin ^{2}(\theta / 2)\right] b_{n}\right\rangle_{b}\right|^{2} d \kappa^{2}
$$

where $\langle A\rangle_{b}=\frac{1}{4 K} \int_{-\theta_{b}}^{\theta_{b}} \frac{A d \theta}{\sqrt{\kappa^{2}-\sin ^{2}(\theta / 2)}}$ denotes bounce average of $A$ over the banana obit, $\kappa^{2}=\left[v^{2} / 2-e \Phi / M-\mu B_{0}(1-\epsilon)\right] /\left(2 \epsilon \mu B_{0}\right)$ is the pitch angle, $\mu=v_{\perp}^{2} /(2 B)$ is the magnetic moment, $\theta_{b}=2 \arcsin (\kappa)$ is the bounce angle of the trapped particles, $I_{1}=\left[\nu_{d} /(2 \epsilon)\right] / I_{0}$ and $I_{0}=\sqrt{\left[\nu_{d} /(2 \epsilon)\right]^{2}+\max \left[\left(n \omega_{d \alpha}\right)^{2}\right]}$, and $\nu_{d}$ is the deflection frequency. For different species, $\nu_{d i}=\nu_{i} x^{-3 / 2} G(x)$ and $\nu_{d e}=\nu_{e} x^{-3 / 2}[1+G(x)]$, where $G(x)=\left[\left(1-\frac{1}{2 x}\right) \operatorname{erf}(\sqrt{x})+\right.$ 
$\left.\frac{1}{\sqrt{\pi} x} e^{-x}\right], \operatorname{erf}(x)$ is the error function, $\nu_{i, e}=\sqrt{2} \pi N_{i} Z_{i}^{4} e_{i, e}^{4} \ln \Lambda /\left[\left(4 \pi \epsilon_{0}\right)^{2} M_{i, e}^{1 / 2} T_{i, e}^{3 / 2}\right]$ are the collisionality of the ions and electrons. $\omega_{d \alpha}=-q \omega_{E}-\omega_{B}$ is the bounce averaged drift frequency, $q \omega_{E} \equiv-q \Phi^{\prime} /\left(\rho B_{0}\right)$ is the $\vec{E} \times \vec{B}$ drift frequency, $\Phi$ is the electric potential, $\omega_{B} \approx \omega_{B 0}[2 E / K-1] x$ is the magnetic drift frequency, $\omega_{B 0}=q^{3} \omega_{t}^{2} /\left(2 \epsilon \Omega_{g}\right)$ is the magnetic drift of the deeply trapped particles with $x=1, x=v^{2} / v_{t}^{2}$ is the normalized energy, and $\Omega_{g}$ is the gyro frequency. $F \equiv 2\left[E / K-1+\kappa^{2}\right]$, and $K\left(\kappa^{2}\right)$ and $E\left(\kappa^{2}\right)$ are the elliptic integrals of the first and second kind, respectively.

When $\nu_{* d} \ll 1$ and $x_{\text {min }} \equiv\left|q \omega_{E} / \omega_{B 0}\right| \gg 1$, the solution of the pitch angle integral can be written as [13],

$$
I_{\kappa n, \nu-\sqrt{\nu}}=\frac{I_{1}^{2}}{\nu_{d} /(2 \epsilon)} \int_{0}^{1} 4 K F\left|n \partial_{\kappa^{2}}\left\langle b_{n}\left(1-e^{-\left(1+i \sigma_{E}\right) y}\right)\right\rangle_{b}\right|^{2} d \kappa^{2}
$$

where $\sigma_{E}= \pm 1$ depending on the sign of the electric field, $y \equiv\left(1-\kappa^{2}\right) / \Delta \kappa^{2}, \Delta \kappa^{2} \approx$ $\sqrt{\frac{8 \nu_{* d}}{\ln \left(16 / \sqrt{8 \nu_{* d}}\right)}}$ is the boundary layer width. The final results do not depend on the sign of $\sigma_{E}$. This regime is the so-called $\nu-\sqrt{\nu}$ regime.

There is a resonance pitch $\kappa_{0}$, at which $\omega_{d \alpha}=0$, when $x>x_{\min } \equiv\left|q \omega_{E} / \omega_{B 0}\right|$. When $\nu_{* d, s b p} \equiv\left[\nu_{d} /(2 \epsilon)\right] /\left|n \frac{d \omega_{d \alpha}}{d \kappa^{2}}\right| \approx\left(x_{m i n} / x\right) \nu_{* d} \ll 1$ and $x>x_{\text {min }}$ the solution of the pitch angle integral can be written as [14],

$$
I_{\kappa n, s b p}=\left.\frac{4 \pi K\left|n\left\langle b_{n}\right\rangle_{b}\right|^{2}}{\left|n d_{\kappa^{2}}\left(\omega_{d \alpha}\right)\right|}\right|_{\kappa^{2}=\kappa_{0}^{2}}
$$

This regime is the so-called superbanana plateau regime.

The smoothly connected formula from the analytic solutions in different regimes [15] can be written as,

$$
I_{\kappa n, C}=H\left(x_{\min }-x\right) \frac{I_{\kappa n, \nu-\sqrt{\nu}}}{1+I_{\kappa n, \nu-\sqrt{\nu}} / I_{\kappa n, 1 / \nu}}+H\left(x-x_{m i n}\right) \frac{I_{\kappa n, s b p}}{1+I_{\kappa n, s b p} / I_{\kappa n, 1 / \nu}}
$$

where $H(x)$ is the Heaviside step function. $H(x)=1$ for $x \geq 0$ and $H(x)=0$ for $x<0$.

Compared to the connected formula in [15], the general solution in the $\nu-\sqrt{\nu}$ regime Eq. (9), rather than the solution in the $\sqrt{\nu}$ regime only, is used in the connected formula. To avoid the singularity for $\nu_{* d} \gg 1$, i.e. for the $1 / \nu$ regime case in the connected formula, it is necessary to use $\nu_{* d} /\left(1+\nu_{* d}\right)$ to replace $\nu_{* d}$ in Eq. (9).

\section{Modelling the NTV torque and comparing it with the observed torque}

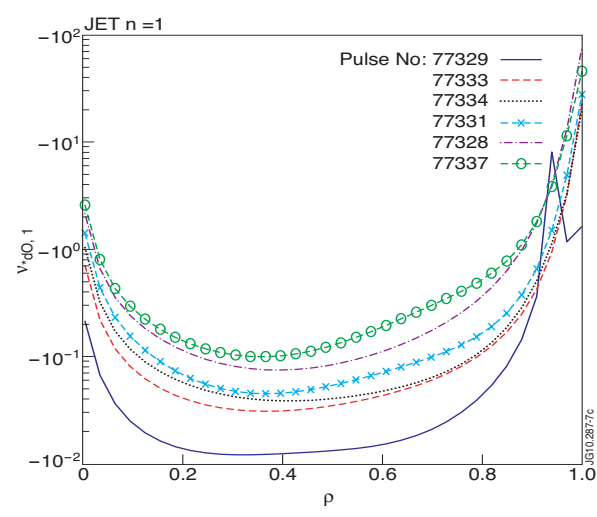

FIG. 7: The profiles of $\nu_{* d 0, i}$

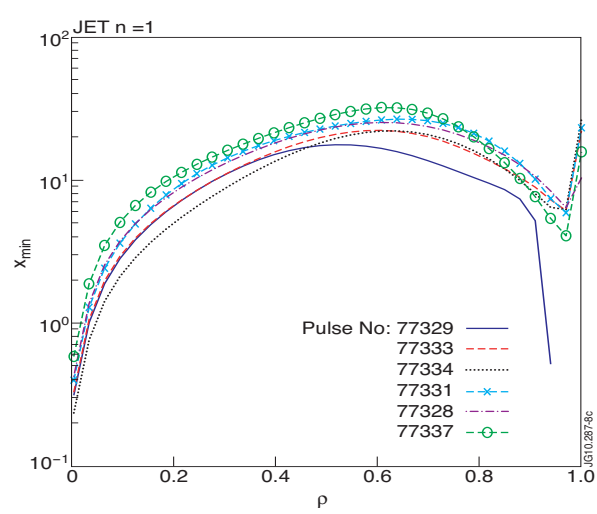

FIG. 8: The profiles of $x_{\text {min }}$ 
The profiles of the $\nu_{* d 0}=\left.\nu_{* d}\right|_{\nu_{d} / \nu=1}$ of ions are shown in Fig. 7. Here, the $\vec{E} \times \vec{B}$ drift frequency is calculated from the radial force balance equation for single ion species, $q \omega_{E}=\omega_{\phi}-q\left(\omega_{\theta}-\omega_{* i}\right)$. The neoclassical value of the poloidal rotation, $\omega_{\theta} \approx k_{n c} \omega_{* T i}$ with $k_{n c}=1.17[23,24]$, is used in this calculation. It shows that $\nu_{* d 0}<1$ almost everywhere. The NTV in the $\nu-\sqrt{\nu}$ and the superbanana regimes are important. The observed collisionality dependence is obviously contradict to the NTV $\nu-\sqrt{\nu}$ regime.

The profiles of $x_{\text {min }}$ are shown in Fig. 8. It shows that $x_{\min }$ is close to 1 in the plasma core region. Therefore, it is close to the transition between the $\nu-\sqrt{\nu}$ and the superbanana plateau regimes. The resonant particles will give a dominant contribution here and causes a different scaling on the collisionality in this range.

The calculated NTV torque density profiles are shown in Fig. 9. Here, the variation of the magnetic field strength Eq. (2) is calculated with the perturbation field $\vec{b}$ calculated from vacuum field assumption. The fundamental harmonic $n=1$ gives the dominant contribution in the NTV torque as discussed before [8]. It shows that the strongest contribution is in the plasma core, which is mainly contributed from the resonant particles. This is consistent with the observations. The comparison of the observed torque and the NTV torque profile for $\rho \in[0.1,0.3]$ is shown in Fig. 10. The NTV torque shows the same tendency as the observed torque. However, the magnitude of the NTV torque is still about 1-2 orders smaller than the observed one.

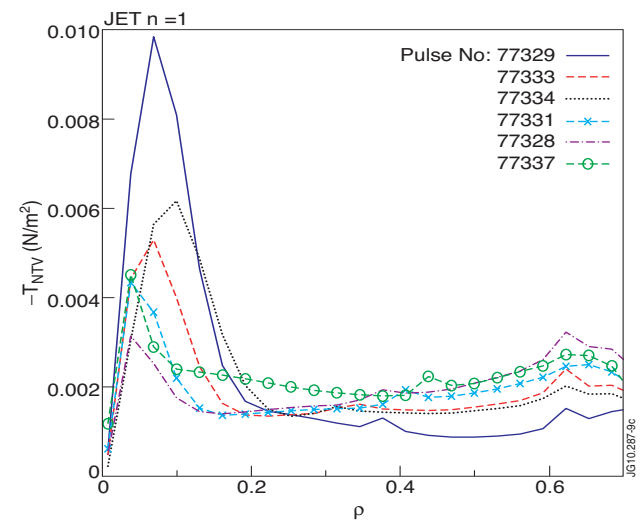

FIG. 9: The profiles of the calculated torque density profiles

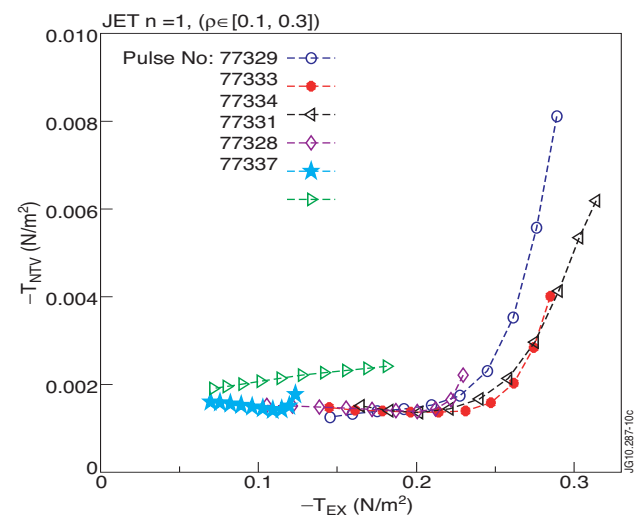

FIG. 10: Comparison between the observed torque and the NTV torque

The calculated NTV torque induced by $m / n=6 / 2$ DED on TEXTOR is also much smaller than that on the JET, because of the great decay of the perturbation field inside the plasma with a similar perturbation field level at the plasma edge in this coil configuration, which is shown in Fig. 6.

\section{Summary.}

Non-resonant magnetic braking effect induced by the NAMP is investigated on JET and TEXTOR. The collionality dependence of the torque induced by the $n=1$ NAMP field is obtained on JET. The observed torque is mainly in the plasma core (normalized $\rho<0.4$ ). It increases with decreasing collisionality. The calculation shows that it is close to the transition between the $\nu-\sqrt{\nu}$ and the superbanana plateau regimes in the plasma core. The NTV torque is modeled by using the smoothly connnected formula in the collisionless regimes. The calculated collisionality dependence has the same tendency as the experimental observation due to the resonant particle effect. The strongest NTV torque is also located in the plasma core because of the resonant particle effect. However, the magnitudes of the NTV torque is still about 1-2 orders smaller than the observed 
ones on JET. The bounce resonance [16, 25] could be important in this case, because the $\vec{E} \times \vec{B}$ drift frequency is close to the bounce frequency of the thermal particles. It will be investigated in the future.

There is no obvious braking effect with $m / n=6 / 2$ DED coils for both resonant and non-resonant pitch before the field penetrates on TEXTOR. The calculated NTV torque on TEXTOR is also very small, because of the great decay of the perturbation field inside the plasma with a similar perturbation field level at the plasma edge in this coil configuration.

The observations on JET and TEXTOR suggest that the non-resonant magnetic braking effect strongly depends on the coil configuration. To avoid the non-resonant magnetic braking, the RMP coil configuration should reduce the non-resonant harmonics and be located as close as possible to the plasma or use a high $\mathrm{n}$ number so that the field rapidly decays inside the plasma.

\section{Acknowledgement.}

This work was supported by EURATOM and carried out within the framework of the European Fusion Development Agreement. The views and opinions expressed herein do not necessarily reflect those of the European Commission.

[1] T. Evans et al., Phys. Rev. Lett. 92, 235003 (2004).

[2] Y. Liang et al., Phys. Rev. Lett. 98, 265004 (2007).

[3] E. J. Strait et al., Phys. Plasmas 11, 2505 (2004).

[4] W. Zhu et al., Phys. Rev. Lett. 96, 225002 (2006).

[5] A.M. Garofalo et al., Phys. Rev. Lett. 101, 195005 (2008).

[6] H. Reimerdes et al., Nucl. Fusion 49, 115001 (2009).

[7] S. A. Sabbagh et al., Nucl. Fusion 50, 025020 (2010).

[8] Y. Sun et al., Plasma Phys. Control. Fusion 52, 105007 (2010).

[9] K. C. Shaing, Phys. Plasmas 10, 1443 (2003).

[10] M. Bécoulet et al., Nucl. Fusion 49, 085011 (2009).

[11] M.-D. Hua et al., Plasma Phys. Control. Fusion 52, 035009 (2010).

[12] Y. Liang et al., Nucl. Fusion 50, 025013 (2010).

[13] K. C. Shaing et al., Phys. Plasmas 15, 082506 (2008).

[14] K. C. Shaing S. A. Sabbagh and M. S. Chu, Plasma Phys. Control. Fusion 51, 035009 (2009).

[15] K. C. Shaing S. A. Sabbagh and M. S. Chu, Nucl. Fusion 50, 025022 (2010).

[16] J.-K. Park A. H. Boozer and J. E. Menard, Phys. Rev. Lett. 102, 065002 (2009).

[17] Y. Sun et al. (to be published in Oct.), Phys. Rev. Lett. 105 (2010).

[18] K. H. Finken et al., Nucl. Fusion 39, 637 (1999).

[19] J.-K. Park et al., Phys. Plasmas 16, 056115 (2009).

[20] K. C. Shaing S. A. Sabbagh and M. S. Chu, Plasma Phys. Control. Fusion 51, 035004 (2009).

[21] K. C. Shaing S. A. Sabbagh and M. S. Chu, Plasma Phys. Control. Fusion 51, 055003 (2009).

[22] A. J. Cole C.C. Hegna and J.D. Callen, Phys. Rev. Lett. 99, 065001 (2007).

[23] S. P. Hirshman and D. J. Sigmar, Nucl. Fusion 21, 1079 (1981).

[24] F. L. Hindon and R. D. Hazeltine, Rev. Mod. Phys. 48, 239 (1976).

[25] K. C. Shaing M. S. Chu and S. A. Sabbagh, Plasma Phys. Control. Fusion 51, 075015 (2009). 